\title{
Visualization of microaneurysms in macular telangiectasia type 1 on optical coherence tomography angiography before and after photocoagulation
}

\author{
Mitsuko Nakai $^{1} \cdot$ Hisashi Iwami ${ }^{1} \cdot$ Hisashi Fukuyama ${ }^{1} \cdot$ Fumi Gomi $^{1}$ (D) \\ Received: 2 June 2020 / Revised: 9 September 2020 / Accepted: 21 September 2020 / Published online: 2 October 2020 \\ (C) The Author(s) 2020
}

\begin{abstract}
Purpose To evaluate changes in the visualization of microaneurysms (MAs) in cases of macular telangiectasia (Mac Tel) type 1 on optical coherence tomography angiography (OCTA) before and after treatment with direct photocoagulation and to evaluate their relationship with treatment efficacy.

Methods The study included 12 eyes from 12 patients ( 8 men, 4 women; mean age 72.1 years) with Mac Tel type 1 accompanied by cystoid macular edema. OCTA for the evaluation of MAs was performed before and $15 \mathrm{~min}$ and 6, 12, and 24 weeks after photocoagulation. The best-corrected visual acuity (BCVA) and central retinal thickness (CRT) were also evaluated.

Results A total of 73 MAs were detected within the areas of macular edema on OCTA, and 39 of these underwent photocoagulation. At $15 \mathrm{~min}$ after treatment, 17 MAs were no longer visible on OCTA. At 6 weeks, two MAs had reappeared, whereas five additional MAs were no longer visible. The CRT in eyes with resolved MA was significantly less than that in eyes with persistent MAs $(p=0.016)$. At 24 weeks, seven eyes had no visible MAs, and the BCVA was not significantly different from baseline. Conclusion OCTA can monitor changes in the visualization of MAs associated with Mac Tel type 1 after direct photocoagulation. Eyes in which MAs disappeared after treatment could recover from cystoid macular edema.
\end{abstract}

Keywords Macular telangiectasia $\cdot$ Optical coherence tomography angiography $\cdot$ Microaneurysms $\cdot$ Photocoagulation

\section{Introduction}

Macular telangiectasia (Mac Tel) is the name for a group of rare diseases that cause spontaneous capillary dilatation within the macula. This condition was first described by Gass and Oyakawa in 1982 as idiopathic juxtafoveal retinal telangiectasia [1]. Yannuzzi et al. later referred to it as idiopathic macular telangiectasia and classified into three types on the basis of optical coherence tomography (OCT) findings [2]. In Japan, Mac Tel type 1, which is characterized by aneurysmal telangiectasia, has a higher prevalence than Mac Tel type 2 [3]. Mac Tel type 1 is more often found in men, is usually unilateral, and is characterized by exudative lesions due to telangiectasia on the temporal side of the macula.

Fumi Gomi

fgomi@hyo-med.ac.jp

1 Department of Ophthalmology, Hyogo College of Medicine, 1-1, Mukogawa, Nishinomiya 663-8501, Japan
Mac Tel type 1 is often encountered in clinical practice, because cystoid macular edema (CME) associated with focal capillary dilatation and microaneurysms (MAs) causes visual deterioration [1,2]. Currently, fluorescein angiography (FA) is necessary to assess the location of lesions in telangiectasia and the severity of leakage. Indocyanine green angiography (ICGA) is also useful in depicting the size, location, and morphology of each lesion, because diffusion of ICGA through the small fenestrations of retinal vessels is limited owing to its protein-bonding nature within the blood [4, 5]. However, FA and ICGA are invasive and time-consuming.

Optical coherence tomography angiography (OCTA) is a recently established technology to visualize retinal-choroidal vessels using OCT principles; it can be performed repeatedly because of its noninvasive nature. OCTA provides more detailed information on retinal vascular abnormalities than OCT [6-10].

To date, there is no proven therapy for Mac Tel; however, anti-vascular endothelial growth factor (VEGF) drugs are sometimes administered to resolve CME, but the 


\section{Key Messages}

- Direct photocoagulation for microaneurysms (MAs) associated with macular telangiectasia type 1 is effective for refractory cystoid macular edema.

- Optical coherence tomography angiography clearly visualized MAs and the corresponding signals promptly disappeared after direct photocoagulation.

- Macular edema reduced in eyes in which MAs disappeared.

improvement is reported to be transient [11]. As in cases of MAs associated with diabetic maculopathy, the basic treatment for persistent CME in Mac Tel type 1 is direct application of photocoagulation to abnormally dilated retinal capillary lesions including MAs $[12,13]$. To increase the success rate, navigated laser photocoagulation using FA [14] and ICGA $[4,5]$ is suggested. However, as an alternative to the use of these invasive forms of angiography, OCTA-guided photocoagulation can be helpful because it can demonstrate prompt changes in blood flow. In this study, we evaluated short-term changes in the visualization of MAs on OCTA in cases of Mac Tel type 1 before and after treatment with focal photocoagulation and further evaluated their relationship with treatment efficacy.

\section{Materials and methods}

This retrospective study enrolled consecutive patients with CME due to Mac Tel type 1 who were diagnosed and treated with photocoagulation from June 2016 to December 2019 at Hyogo College of Medicine. The Institutional Review Board of Hyogo College of Medicine approved this study (No. 2426), which followed the tenets of the Declaration of Helsinki. Informed consent was obtained from all subjects.

Patients were comprehensively diagnosed with Mac Tel type 1 according to their medical history, FA, and ICGA findings, and the condition of the fellow eye. Patients with neovascular maculopathies (e.g., age-related macular degeneration, polypoidal choroidal vasculopathy, and retinal angiomatous proliferation) or other retinal vascular diseases such as diabetic maculopathy and retinal vein occlusion, were excluded.

The treatment criteria for direct photocoagulation were that MAs (1) were visible on FA, ICGA, and OCTA, (2) visible leakage on FA associated with persistent or refractory CME involving the fovea, (3) had a size of $50 \mu \mathrm{m}$ or more (larger than the size of the laser spot, to avoid damage to surrounding tissue), and (4) did not show involvement within the foveal avascular zone. After pupillary dilation and instillation of topical anesthesia, photocoagulation at a wavelength of $577 \mathrm{~nm}$
(NIDEK, MC-500, Tokyo, Japan) was performed by two retina specialists ( $\mathrm{FG}$ and $\mathrm{HI}$ ). The laser parameters were as follows: (1) spot size of 50-100 $\mu \mathrm{m}$; (2) pulse duration of $0.05-0.1 \mathrm{~s}$; (3) single spot; and (4) burn intensity of 80-140 $\mathrm{mW}$, so that only the lesions of the MAs became gray or white. A combined anti-VEGF drug injection was administered at the discretion of the physician.

All patients underwent ophthalmological examinations including best-corrected visual acuity (BCVA), fundus examination, OCT, and OCTA (RS-3000, NIDEK, Japan, or DRI OCT Triton, Topcon, Japan) before and after treatment. The OCTA examination used a $3.0 \times 3.0$ or $4.5 \times 4.5-\mathrm{mm}$ square area and was performed before and just after photocoagulation (within $15 \mathrm{~min}$ ), and around 6,12, and 24 weeks thereafter. The same OCTA device was used repeatedly for the examination of each given patient, and default segmentation slabs of the superficial and deep retina were used in each device. The number and visibility of MAs in each slab of the superficial and deep retina on enface OCTA within the 3-mm field of the Early Treatment of Diabetic Retinopathy Study (ETDRS) circle were independently evaluated for each time point by two examiners (MN and HI), without reference to FA or ICGA. If the interpretations differed, a third observer (FG) interpreted the results. Then, the number of MAs on the baseline OCTA was compared with that at the corresponding area on FA or ICGA. Additional treatment was administered between 6 and 24 weeks, according to the physician's decision.

The decimal BCVA, obtained using a Landolt $\mathrm{C}$ chart, was converted to a logarithm of minimum angle resolution (logMAR). The changes in BCVA and central retina thickness (CRT) were also compared before and after the treatment.

\section{Statistical analysis}

All statistical analyses were performed using EZR software (Saitama Medical Center, Jichi Medical University). Continuous variables are expressed as the mean \pm standard deviation (SD). The Wilcoxon signed-rank test and Friedman test were used to compare the microaneurysm 


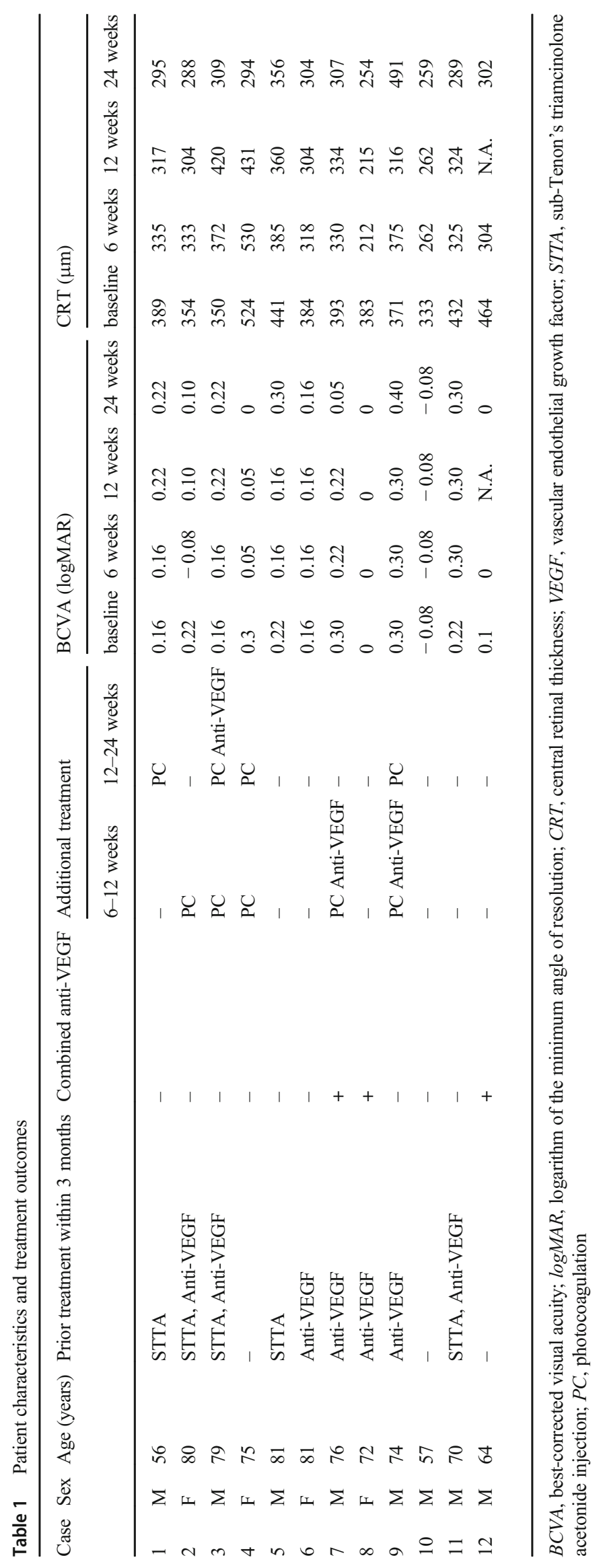


Table 2 Visualization of microaneurysms on optical coherence tomography angiography

\begin{tabular}{|c|c|c|c|c|c|c|c|c|c|c|c|c|c|c|c|c|}
\hline \multirow[t]{2}{*}{ Case } & \multirow[t]{2}{*}{ MAs on FA $(n)$} & \multicolumn{3}{|c|}{$\begin{array}{l}\text { MAs on baseline } \\
\text { OCTA }(n)\end{array}$} & \multicolumn{3}{|c|}{$\begin{array}{l}\text { MAs on OCTA } \\
\text { at } 15 \min (n)\end{array}$} & \multicolumn{3}{|c|}{$\begin{array}{l}\text { MAs on OCTA } \\
\text { at } 6 \text { weeks }(n)\end{array}$} & \multicolumn{3}{|c|}{$\begin{array}{l}\text { MAs on OCTA } \\
\text { at } 12 \text { weeks }(n)\end{array}$} & \multicolumn{3}{|c|}{$\begin{array}{l}\text { OCTA at } 24 \text { weeks, } \\
\text { visible MAs }(n)\end{array}$} \\
\hline & & $S$ & $D$ & Both & $S$ & $D$ & Both & $S$ & $D$ & Both & $S$ & $D$ & Both & $S$ & $D$ & Both \\
\hline 1 & 17 & 2 & 13 & 2 & 1 & 7 & 1 & 1 & 8 & 1 & 1 & 8 & 1 & 1 & 8 & 1 \\
\hline 2 & 4 & 1 & 2 & 1 & 0 & 1 & 0 & 0 & 2 (new 1) & 0 & 0 & 2 (new 1) & 0 & 0 & 2 (new 1) & 0 \\
\hline 3 & 3 & 0 & 2 & 0 & 0 & 2 & 0 & 0 & 2 & 0 & 0 & 2 & 0 & 0 & 0 & 0 \\
\hline 4 & 30 & 12 & 13 & 5 & 5 & 8 & 2 & 1 & 5 & 1 & 1 & 5 & 1 & 1 & 5 & 1 \\
\hline 5 & 2 & 1 & 3 & 1 & 0 & 1 & 0 & 0 & 1 & 0 & 0 & 0 & 0 & 0 & 0 & 0 \\
\hline 6 & 1 & 1 & 4 & 1 & 0 & 3 & 0 & 0 & 3 & 0 & 0 & 3 & 0 & 0 & 3 & 0 \\
\hline 7 & 4 & 0 & 4 & 0 & N.A. & N.A. & N.A. & 0 & 2 & 0 & 0 & 2 & 0 & 0 & 0 & 0 \\
\hline 8 & 3 & 0 & 2 & 0 & 0 & 1 & 0 & 0 & 0 & 0 & 0 & 0 & 0 & 0 & 0 & 0 \\
\hline 9 & 8 & 0 & 5 & 0 & 0 & 3 & 0 & 0 & 3 (new 1) & 0 & 0 & 3 (new 1) & 0 & 2 (new 2) & 3 (new 2) & 2 \\
\hline 10 & 1 & 0 & 1 & 0 & 0 & 1 & 0 & 0 & 0 & 0 & 0 & 0 & 0 & 0 & 0 & 0 \\
\hline 11 & 2 & 2 & 5 & 2 & 0 & 3 & 0 & 0 & 0 & 0 & 0 & 0 & 0 & 0 & 0 & 0 \\
\hline 12 & 21 & 6 & 11 & 5 & 5 & 10 & 5 & 4 & 9 & 4 & N.A. & N.A. & N.A. & 4 (new 1) & 10 (new 3) & 4 \\
\hline
\end{tabular}

MAs, microaneurysms; OCTA, optical coherence tomography angiography; $S$, superficial retinal slab; $D$, deep retinal slab; $N . A$., unable to analyze because of poor image quality

lesions, BCVA, and CRT before and after photocoagulation. $P$ values of $<0.05$ were considered statistically significant.

\section{Results}

A total of 12 eyes in 12 patients ( 8 men and 4 women) were included in the study (Table 1). The mean age of the patients was $72.1 \pm 8.4$ years (range, $56-81$ years). One or more lesions of telangiectasia and MAs were detected on FA and OCTA. ICGA was obtained in 11 patients. Nine eyes (75\%) had a history of treatment with anti-VEGF drugs and/or sub-
Tenon's triamcinolone acetonide injection within 3 months, but CME remained.

At baseline, the mean $\log$ MAR BCVA was $0.17 \pm 0.11$ (range, -0.08 to 0.30 ). All patients complained of blurred vision and metamorphopsia associated with the CME. The CRT values averaged $401.50 \pm 52.29 \mu \mathrm{m}$ (range, 333$524 \mu \mathrm{m})$.

The numbers of MAs on FA and OCTA are shown in Table 2. On OCTA, microvascular abnormalities consisting of tortuous capillaries and MAs were found in the superficial and deep retinal slabs in all eyes. A total of 73 MAs were visualized on OCTA; 65 MAs in the deep retinal slab and 25 MAs in the superficial retinal slab. Seventeen relatively

Table 3 Changes in number of microaneurysms with photocoagulation

\begin{tabular}{llllll}
\hline Case & $\begin{array}{l}\text { Photocoagulated } \\
\text { MAs }(n)\end{array}$ & $\begin{array}{l}\text { Persistent } \\
\text { MAs at 15 min }(n)\end{array}$ & $\begin{array}{l}\text { Persistent } \\
\text { MAs at 6 weeks }(n)\end{array}$ & $\begin{array}{l}\text { Persistent } \\
\text { MAs at 12 weeks }(n)\end{array}$ & $\begin{array}{l}\text { Persistent } \\
\text { MAs at 24 weeks }(n)\end{array}$ \\
\hline 1 & 2 & 1 & 1 & 1 & 1 \\
2 & 1 & 0 & 1 & 1 & 1 \\
3 & 2 & 2 & 2 & 2 & 0 \\
4 & 16 & 7 & 5 & 5 & 5 \\
5 & 1 & 0 & 0 & 0 & 0 \\
6 & 1 & 0 & 0 & 0 & 0 \\
7 & 2 & N.A. & 0 & 0 & 0 \\
8 & 1 & 1 & 0 & 0 & 0 \\
9 & 3 & 2 & 2 & 1 & 1 \\
10 & 1 & 1 & 0 & 0 & 0 \\
11 & 2 & 0 & 0 & 0 & 0 \\
12 & 7 & 6 & 5 & N.A. \\
\hline
\end{tabular}

MAs, microaneurysms; N.A., unable to analyze because of poor image quality 

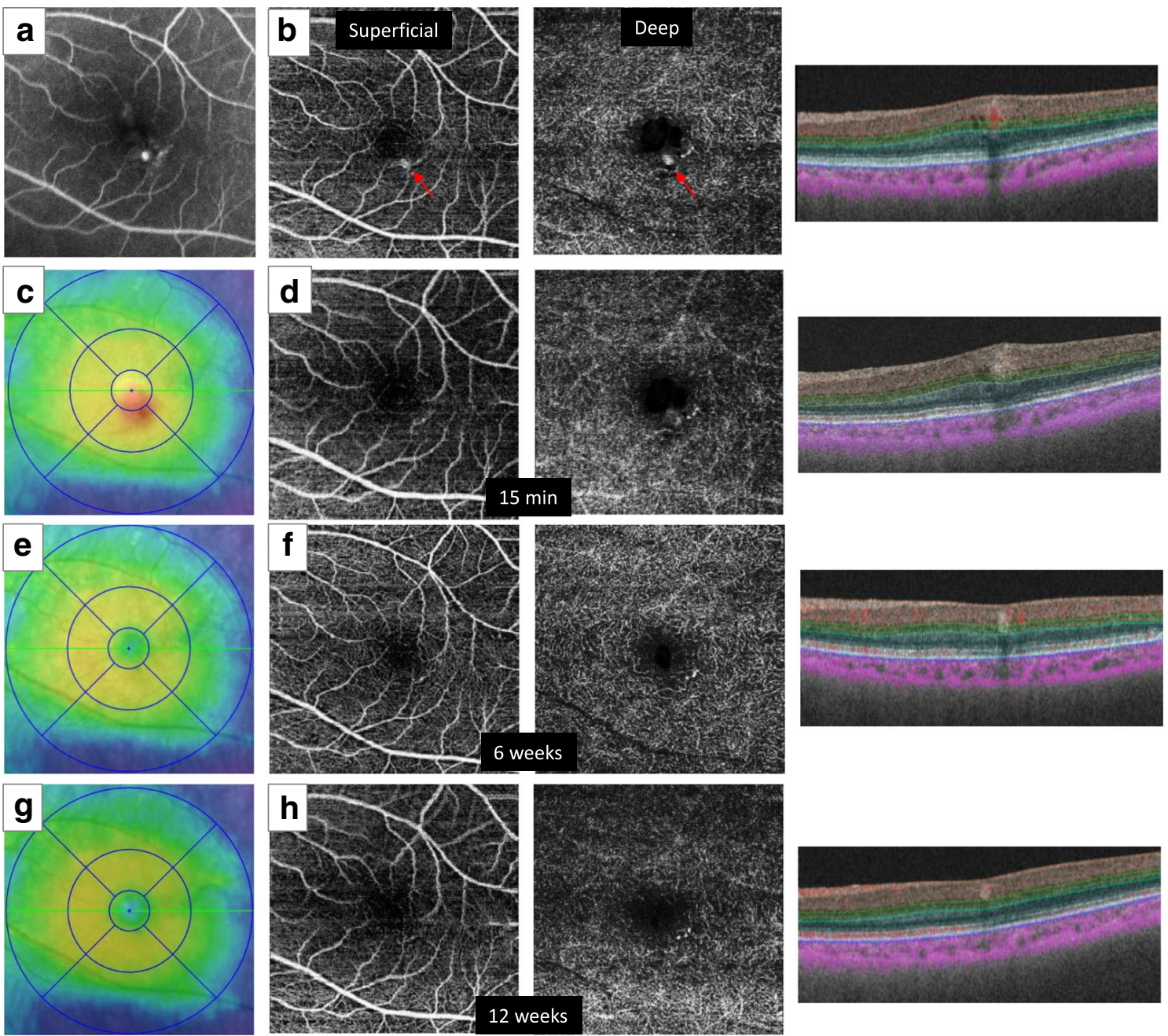

Fig. 1 Images from the left eye of case 6. a Fluorescein angiography showed retinal microaneurysms (MAs). b Baseline enface and B scan optical coherence tomography angiography (OCTA) showed hyperreflective signals corresponding to MAs that were segmented into both the superficial and deep layers. One MA indicated with a red arrow was treated with photocoagulation. c Baseline OCT map showing a

large MAs were seen in both superficial and deep retinal layers, whereas eight MAs were only seen in the superficial layer. Compared with the images on FA and ICGA, the visibility of MAs on OCTA was inferior, and some MAs were not detectable. However, some hyperreflective dots seen on OCTA were derived from hard exudates.

Direct photocoagulation was administered to 39 MAs meeting the treatment criteria. The anti-VEGF drug bevacizumab was injected into three eyes (cases 7,8 , and 12 ) at the time of photocoagulation.

The changes in the numbers of MAs depicted on OCTA after treatment are shown in Tables 2 and 3. At 15 min after photocoagulation, 17 of the MAs (45.9\%), most of which were seen in both superficial and deep layers, could not be visualized on OCTA. Five MAs had disappeared by 6 weeks. Conversely, two MAs that were not visible at $15 \mathrm{~min}$ region of cystoid macular edema. d OCTA at 15 min showed no apparent signals within the treated MA region. e An OCT map at 6 weeks showed resolution of macular edema. $\mathbf{f}$ OCTA showed no signals within the treated MA. g An OCT map at 12 weeks showed no recurrence of edema. h OCTA also showed no recurrence of hyperreflective signals, and untreated small MAs were visible

reappeared on 6 weeks. At this time, treated MAs were not visible in six (50\%) eyes. Between 6 and 24 weeks, additional treatments including photocoagulation and anti-VEGF were performed in 6 eyes (Table 1). At 24 weeks, 13 of the 39 initially photocoagulated MAs $(33.3 \%)$ were persistent. After 24 weeks, three eyes had new MAs and five eyes (cases 1, 2, 4, 9, and 12) had visible MAs on OCTA. Findings from representative cases are shown in Figs. 1 and 2.

The mean CRT of $340.08 \pm 73.61 \mu \mathrm{m}$ at 6 weeks was significantly less than that of $401.50 \pm 52.29 \mu \mathrm{m}$ at baseline $(p=$ 0.0068 ). Except for three eyes with initially combined antiVEGF therapy, the CRT in eyes with resolved MAs (cases 5, 6,10 , and 11) was significantly less than that in eyes with persistent MAs (cases 1, 2, 3, 4, and 9; $p=0.016$ ). From 6 to 24 weeks, one additional anti-VEGF injection was administered in three eyes. Seven eyes had a foveal pit without a cyst 

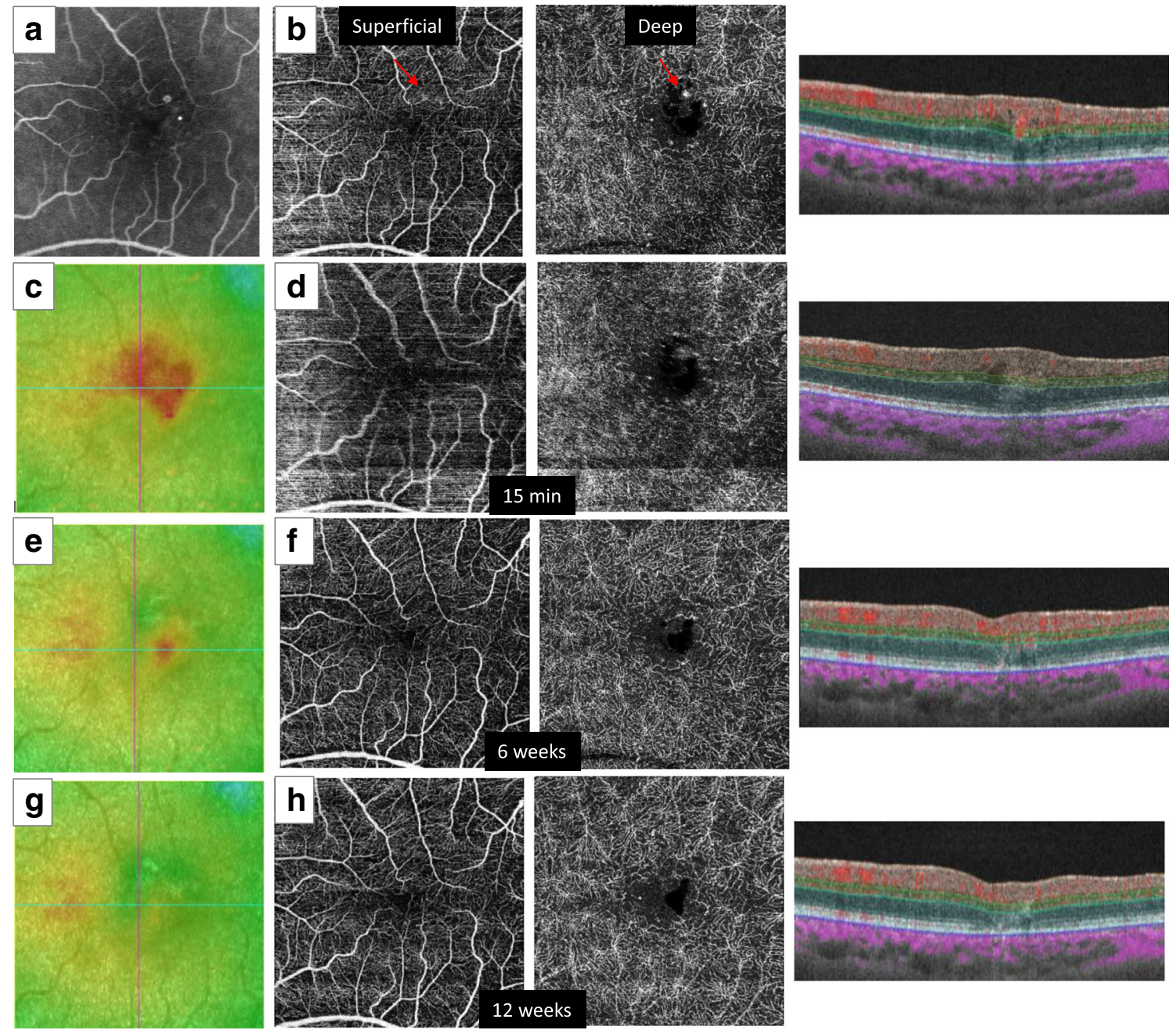

Fig. 2 Images from the left eye of case 5. a Fluorescein angiography showed two retinal microaneurysms (MAs). b Baseline enface and B scan optical coherence tomography angiography (OCTA) showed hyperreflective signals corresponding to MAs that were segmented into both the superficial and deep layers. One MA indicated with a red arrow was treated with photocoagulation. c Baseline OCT map showing a region of cystoid macular edema. d OCTA at 15 min showed no

and four eyes (cases 3, 4, 7, and 12) had a foveal pit with slight cystic changes, while the remaining eye (case 9) did not show a significant reduction in CRT. The mean BCVA was $0.11 \pm$ $0.13 \log$ MAR (range, -0.08 to $0.30 \log$ MAR) at 6 weeks, and $0.14 \pm 0.14 \log$ MAR (range, -0.08 to $0.30 \log$ MAR) at 24 weeks. These values were not significantly different from the baseline values ( $p=0.12$ and $p=0.48$, respectively). At 24 weeks, no eyes developed retinal pigment epithelial atrophy, but two eyes showed a thinning of the outer retinal layer around the area of photocoagulation.

\section{Discussion}

In this study, we could detect visible changes to MAs on OCTA before and after focal photocoagulation. Before hyperreflective signals within the treated MA region. e An OCT map at 6 weeks showed reduction of macular edema. f OCTA showed no apparent signals within the treated MA. $g$ An OCT map at 12 weeks showed no retinal edema. $\mathbf{h}$ OCTA also showed no recurrence of hyperreflective signals and untreated small MAs were visible. B scan OCTA showed slight disruption of the outer retinal layer

treatment, MAs were seen preferentially in the deep retinal slab, as Matte et al. [15] reported previously, but relatively large MAs were found in both the superficial and deep slabs. Spaide et al. [16] also reported that some MAs in patients with diabetic retinopathy were seen in both the superficial and deep retinal slabs. However, as reported previously [7, 17, 18], not all MAs observed on FA or ICGA were delineated on OCTA. As we did not perform manual segmentation, some MAs could not be visualized because of errors in segmentation caused by retinal edema; repeated scans [19] and multiple image averaging [20] might increase the ability to detect MAs. Hard exudates were found to be the major cause of artifacts and were difficult to discriminate from MAs.

After photocoagulation, almost half of the treated MAs disappeared from OCTA within $15 \mathrm{~min}$. All but two of the MAs that immediately disappeared were not apparent at 6 weeks and 
later. Furthermore, a few MAs that were visible just after photocoagulation were not visible at 6 weeks or later. Previously, in an analysis of FA, Sachdev et al. [21] observed that MA closure was only $0.67 \%$ at 2 weeks, but increased to $89.6 \%$ by 12 weeks. Using spectral-domain OCT, Lee et al. [22] and Yamada et al. [23] reported that MAs associated with diabetic maculopathy and that closed following focal laser photocoagulation showed uniform hyperreflectivity, finally disappearing over time. Immediately after photocoagulation, the lumen of MAs is changed and turbulent blood flow may occur within the treated MAs, making the MAs invisible on OCTA, as Nakao et al. reported. [24] Later, thrombus is formed, and the obstruction of blood flow within vessels can cause permanent closure of MAs. These time- and efficacy-dependent changes within the MAs after photocoagulation could cause a change in visibility on OCTA during the follow-up period.

Earlier disappearance of signals corresponding to the MAs on OCTA suggests the success of photocoagulation, although complete closure of MAs requires a long time, and in some cases, recanalization may occur during follow-up. In our study, the eyes in which MAs disappeared showed significant reductions in CRT, although 7 of 12 eyes with persistent MAs and CME required additional treatment over 24 weeks. Finally, all but one eye obtained a foveal pit. To avoid laserinduced damage, we selected the MAs to treat, and the results suggest that the resolution of CME depends on which MAs cause CME, and whether they are treatable with photocoagulation. Hirano et al. [25] reported that a combined focal/grid laser successfully reduced the number of anti-VEGF injections for diabetic macular edema. Therefore, we suggest that careful evaluation of OCTA images for MAs could reduce additional treatments with anti-VAGF and photocoagulation.

Regarding the BCVA, it had not improved significantly from baseline, although the CRT decreased significantly. A previous report showed that, in addition to the CRT, microvascular density, ellipsoid zone disruption, and the presence of disorganization in the retinal inner slabs might be predictive biomarkers of VA in Mac Tel type 1 [26]. We did not observe any retinal pigment epithelial atrophy during the posttreatment period, and only two eyes showed a thinning of the outer retinal layer around the area of photocoagulation; therefore, for photocoagulation to achieve its optimum effectiveness, a careful procedure targeting only selected MAs may be essential.

The limitations of this study are the small sample size and relatively short observation period. As mentioned earlier, the number of MAs could be inaccurate because of segmentation errors and artifacts. Furthermore, the use of combined antiVEGF drugs depended on the physician.

In conclusion, the visualization of MAs on OCTA changed after direct photocoagulation. The results suggest that the success of photocoagulation can be monitored using noninvasive OCTA.
Author's contributions Not applicable.

Data availability Not applicable.

\section{Compliance with ethical standards}

Conflict of interest The authors declare that they have no conflict of interest.

Ethical approval The Institutional Review Board of Hyogo College of Medicine approved this study (No. 2426), which followed the tenets of the Declaration of Helsinki.

Informed consent Informed consent was obtained from all individual participants included in the study.

Code availability Not applicable.

Open Access This article is licensed under a Creative Commons Attribution 4.0 International License, which permits use, sharing, adaptation, distribution and reproduction in any medium or format, as long as you give appropriate credit to the original author(s) and the source, provide a link to the Creative Commons licence, and indicate if changes were made. The images or other third party material in this article are included in the article's Creative Commons licence, unless indicated otherwise in a credit line to the material. If material is not included in the article's Creative Commons licence and your intended use is not permitted by statutory regulation or exceeds the permitted use, you will need to obtain permission directly from the copyright holder. To view a copy of this licence, visit http://creativecommons.org/licenses/by/4.0/.

\section{References}

1. Gass JD, Oyakawa RT (1982) Idiopathic juxtafoveolar retinal telangiectasis. JAMA Ophthalmology 100:769-780. https://doi.org/ 10.1001/archopht.1982.01030030773010

2. Yannuzzi LA, Bardal AMC, Freund KB et al (2006) Idiopathic macular telangiectasia. JAMA Ophthalmology 124:450-460. https://doi.org/10.1001/archopht.124.4.450

3. Maruko I, Iida T, Sugano Y et al (2012) Demographic features of idiopathic macular telangiectasia in Japanese patients. Jpn J Ophthalmol 56:152-158. https://doi.org/10.1007/s10384-0110112-5

4. Ueda T, Gomi F, Suzuki M et al (2012) Usefulness of indocyanine green angiography to depict the distant retinal vascular anomalies associated with branch retinal vein occlusion causing serous macular detachment. Retina 32:308-313. https://doi.org/10.1097/IAE. 0b013e31821c40a9

5. Ogura S, Yasukawa T, Kato A et al (2015) Indocyanine green angiography-guided focal laser photocoagulation for diabetic macular edema. Ophthalmologica 234:139-150. https://doi.org/10. $1159 / 000437360$

6. Ishibazawa A, Nagaoka T, Takahashi A et al (2015) Optical coherence tomography angiography in diabetic retinopathy: a prospective pilot study. Am J Ophthalmol 160:35-44.e1. https://doi.org/10. 1016/j.ajo.2015.04.021

7. Hamada M, Ohkoshi K, Inagaki K et al (2018) Correction to: Visualization of microaneurysms using optical coherence tomography angiography: comparison of OCTA en face, OCT B-scan, OCT en face, FA, and IA images. Jpn J Ophthalmol 62:176-178. https://doi.org/10.1007/s10384-018-0577-6 
8. Mané V, Dupas B, Gaudric A et al (2016) Correlation between cystoid spaces in chronic diabetic macular edema and capillary nonperfusion detected by optical coherence tomography angiography. Retina 36:S102-S110. https://doi.org/10.1097/IAE. 0000000000001289

9. Hasegawa N, Nozaki M, Takase N et al (2016) New insights into microaneurysms in the deep capillary plexus detected by optical coherence tomography angiography in diabetic macular edema. Investig Ophthalmol Vis Sci 57:OCT348-OCT355. https://doi. org/10.1167/iovs. 15-18782

10. Matsunaga DR, Yi JJ, De Koo LO et al (2015) Optical coherence tomography angiography of diabetic retinopathy in human subjects. Ophthalmic Surg Lasers Imaging Retina 46:796-805. https://doi. org/10.3928/23258160-20150909-03

11. Demir G, Artunay Ö, Sucu ME et al (2019) Treatment of intravitreal bevacizumab combined with focal laser photocoagulation in the case of macular telangiectasia type 2 with retinal arterial macroaneurysm. Lasers Med Sci 34:427-430. https://doi.org/10. 1007/s10103-018-2587-5

12. Takayama K, Ooto $\mathrm{S}$, Tamura $\mathrm{H}$ et al (2010) Intravitreal bevacizumab for type 1 idiopathic macular telangiectasia. Eye 24: 1492-1497. https://doi.org/10.1038/eye.2010.61

13. Chatziralli IP, Sharma PK, Sivaprasad S (2017) Treatment modalities for idiopathic macular telangiectasia: an evidence-based systematic review of the literature. Semin Ophthalmol 32:384-394. https://doi.org/10.3109/08820538.2015.1096399

14. Kozak I, Oster SF, Cortes MA et al (2011) Clinical evaluation and treatment accuracy in diabetic macular edema using navigated laser photocoagulator NAVILAS. Ophthalmology 118:1119-1124. https://doi.org/10.1016/j.ophtha.2010.10.007

15. Matet A, Daruich A, Dirani A et al (2016) Macular telangiectasia type 1: capillary density and microvascular abnormalities assessed by optical coherence tomography angiography. Am J Ophthalmol 167:18-30. https://doi.org/10.1016/j.ajo.2016.04.005

16. Spaide RF, Klancnik JM, Cooney MJ (2015) Retinal vascular layers imaged by fluorescein angiography and optical coherence tomography angiography. JAMA Ophthalmology 133:45-50. https://doi.org/10.1001/jamaophthalmol.2014.3616

17. Couturier A, Mané V, Bonnin S et al (2015) Capillary plexus anomalies in diabetic retinopathy on optical coherence tomography angiography. Retina 35:2384-2391. https://doi.org/10.1097/IAE. 0000000000000859
18. Miwa Y, Murakami T, Suzuma K et al (2016) Relationship between functional and structural changes in diabetic vessels in optical coherence tomography angiography. Sci Rep 6:1-12. https://doi.org/ 10.1038/srep29064

19. Spaide RF, Fujimoto JG, Waheed NK (2015) Image artifacts in optical coherence tomography angiography. Retina 35:21632180. https://doi.org/10.1097/IAE.0000000000000765

20. Kaizu Y, Nakao S, Wada I et al (2020) Microaneurysm imaging using multiple en face OCT angiography image averaging: morphology and visualization. Ophthalmology Retina 4:175-186. https://doi.org/10.1016/J.ORET.2019.09.010

21. Sachdev N, Gupta V, Abhiramamurthy V et al (2008) Correlation between microaneurysm closure rate and reduction in macular thickness following laser photocoagulation of diabetic macular edema. Eye 22:975-977. https://doi.org/10.1038/sj.eye.6702801

22. Lee SN, Chhablani J, Chan CK et al (2013) Characterization of microaneurysm closure after focal laser photocoagulation in diabetic macular edema. Am J Ophthalmol 155:905-912. https://doi.org/ 10.1016/j.ajo.2012.12.005

23. Yamada Y, Suzuma K, Fujikawa A et al (2013) Imaging of laserphotocoagulated diabetic microaneurysm with spectral domain optical coherence tomography. Retina 33:726-731. https://doi.org/10. 1097/IAE.0b013e3182753960

24. Nakao S, Kaizu Y, Oshima Y et al (2016) Optical coherence tomography angiography for detecting choroidal neovascularization secondary to punctate inner choroidopathy. Ophthalmic Surg Lasers Imaging Retina 47:1157-1161. https://doi.org/10.3928/ 23258160-20161130-13

25. Hirano T, Toriyama $Y$, Iesato $Y$ et al (2017) Effect of leaking perifoveal microaneurysms on resolution of diabetic macular edema treated by combination therapy using anti-vascular endothelial growth factor and short pulse focal/grid laser photocoagulation. Jpn J Ophthalmol 61:51-60. https://doi.org/10.1007/s10384-0160483-8

26. Guo J, Tang W, Ye X et al (2018) Predictive multi-imaging biomarkers relevant for visual acuity in idiopathic macular telangiectasis type 1. BMC Ophthalmol 18:1-7. https://doi.org/10.1186/ s12886-018-0737-y

Publisher's note Springer Nature remains neutral with regard to jurisdictional claims in published maps and institutional affiliations. 